\title{
Recurrence Relations for Strongly $q$-Log-Convex Polynomials
}

\author{
William Y. C. Chen ${ }^{1}$, Larry X.W. Wang ${ }^{2}$ and Arthur L. B. Yang ${ }^{3}$ \\ Center for Combinatorics, LPMC-TJKLC \\ Nankai University, Tianjin 300071, P. R. China \\ Email: ${ }^{1}$ chen@nankai.edu.cn, ${ }^{2}$ wxw@cfc.nankai.edu.cn, \\ 3 yang@nankai .edu . cn
}

\begin{abstract}
We consider a class of strongly $q$-log-convex polynomials based on a triangular recurrence relation with linear coefficients, and we show that the Bell polynomials, the Bessel polynomials, the Ramanujan polynomials and the Dowling polynomials are strongly $q$-log-convex. We also prove that the Bessel transformation preserves log-convexity.

Keywords: log-concave, $q$-log-convexity, strong $q$-log-convexity, Bell polynomials, Bessel polynomials, Ramanujan polynomials, Dowling polynomials
\end{abstract}

AMS Classification: 05A20, 05E99

\section{Introduction}

The main objective of this paper is to study the $q$-log-convexity of a class of polynomials whose coefficients satisfy a triangular recurrence relation with linear coefficients. The notion of log-convexity is closely related to logconcavity. Stanley introduced the concept of $q$-log-concavity, which naturally leads to the notion of $q$-log-convexity. Compared with $q$-log-concave polynomials, $q$-log-convex polynomials did not draw much attention. Only till recently, Liu and Wang [16] have shown that some classical polynomials are $q$-log-convex, such as the Bell polynomials and the Eulerian polynomials.

In this paper, we will show that a polynomial

$$
P_{n}(q)=\sum_{k=0}^{n} T(n, k) q^{k}
$$

is $q$-log-convex if the coefficients $T(n, k)$ satisfy certain recurrence relation with linear coefficients in $n$ and $k$. The concept of strong $q$-log-concavity is due to Sagan [18]. In this framework, we will show that the Bell polynomials, the Bessel polynomials, the Ramanujan polynomials and the Dowling polynomials are strongly $q$-log-convex. 
Let us give a brief review of the background and terminology. Unimodal and log-concave sequences and polynomials often arise in combinatorics, algebra and geometry; See the surveys of Brenti [4, 5] and Stanley [20]. A sequence $\left\{z_{k}\right\}_{k \geq 0}$ of nonnegative real numbers is said to be unimodal if there exists an integer $r \geq 0$ such that

$$
z_{0} \leq z_{1} \leq \cdots \leq z_{r} \geq z_{r+1} \geq z_{r+2} \geq \cdots
$$

It is said to be log-concave if

$$
z_{m}^{2} \geq z_{m+1} z_{m-1}, \quad m \geq 1
$$

and it is said to be strongly log-concave if

$$
z_{m} z_{n} \geq z_{m-1} z_{n+1}, \quad n \geq m \geq 1 \text {. }
$$

For a sequence of positive real numbers, log-concavity is equivalent to strong log-concavity and implies unimodality.

Analogously, a sequence $\left\{z_{k}\right\}_{k \geq 0}$ of nonnegative real numbers is said to be log-convex if

$$
z_{m}^{2} \leq z_{m+1} z_{m-1}, \quad m \geq 1,
$$

and it is said to be strongly log-convex if

$$
z_{m} z_{n} \leq z_{m-1} z_{n+1}, \quad n \geq m \geq 1
$$

It is also easily seen that, for a sequence of positive real numbers, logconcavity is equivalent to the log-convexity of the sequence of the reciprocals; See [16].

However, the equivalence of log-concavity and strong log-concavity does not apply to polynomial sequences. The $q$-log concavity of polynomials has been extensively studied; See, for example, Butler [7], Krattenthaler [14], Leroux [17], and Sagan [18]. Adopting the notation of Sagan [18], we write

$$
f(q) \geq_{q} g(q)
$$

if the difference $f(q)-g(q)$ has nonnegative coefficients as a polynomial of $q$. A sequence of polynomials $\left\{f_{k}(q)\right\}_{k \geq 0}$ over the field of real numbers is called $q$-log-concave if

$$
f_{m}(q)^{2} \geq_{q} f_{m+1}(q) f_{m-1}(q), \quad m \geq 1,
$$

and it is strongly $q$-log-concave if

$$
f_{m}(q) f_{n}(q) \geq_{q} f_{m-1}(q) f_{n+1}(q), \quad n \geq m \geq 1 .
$$


Based on the $q$-log concavity, it is natural to define the $q$-log-convexity and the strong $q$-log-convexity. We say that a polynomial sequence $\left\{f_{n}(q)\right\}_{n \geq 0}$ is $q$-log-convex if

$$
f_{m+1}(q) f_{m-1}(q) \geq_{q} f_{m}(q)^{2}, \quad m \geq 1,
$$

and it is strongly $q$-log-convex if

$$
f_{m-1}(q) f_{n+1}(q) \geq_{q} f_{m}(q) f_{n}(q), \quad n \geq m \geq 1 .
$$

For a sequence of polynomials, $q$-log-convexity is also not equivalent to strong $q$-log-convexity. Note that Butler and Flanigan [8] defined a different $q$ analogue of log-convexity.

For a $q$-log-convex sequence of polynomials $P_{n}(q)$ as given in (1.1), we will be concerned with the linear transformation associated with $P_{n}(q)$ which transforms a sequence $\left\{z_{n}\right\}_{n \geq 0}$ into a sequence $\left\{w_{n}\right\}_{n \geq 0}$ given by

$$
w_{n}=\sum_{k=0}^{n} T(n, k) z_{k} .
$$

We say that the linear transformation preserves log-convexity, if, for any given $\log$-convex sequence $\left\{z_{n}\right\}_{n \geq 0}$ of positive real numbers, the sequence $\left\{w_{n}\right\}_{n \geq 0}$ defined by the above transformation is also log-convex. For the Bell polynomials, the corresponding linear transformation is defined by the Stirling numbers of the second kind. It has been proved that the Bell transformation preserves log-convexity [16]. In this paper, we will show that the Bessel transformation preserves log-convexity.

\section{The strong $q$-log-convexity}

In this section, we consider polynomials

$$
P_{n}(q)=\sum_{k=0}^{n} T(n, k) q^{k}, \quad n \geq 0,
$$

where the coefficients $T(n, k)$ are nonnegative real numbers and satisfy the following recurrence relation

$$
\begin{aligned}
T(n, k)=( & \left.a_{1} n+a_{2} k+a_{3}\right) T(n-1, k) \\
& +\left(b_{1} n+b_{2} k+b_{3}\right) T(n-1, k-1), \text { for } n \geq k \geq 1,
\end{aligned}
$$

and the boundary conditions

$$
T(n,-1)=T(n, n+1)=0, \text { for } n \geq 1,
$$




$$
a_{1} \geq 0, \quad a_{1}+a_{2} \geq 0, \quad a_{1}+a_{2}+a_{3}>0,
$$

and

$$
b_{1} \geq 0, \quad b_{1}+b_{2} \geq 0, \quad b_{1}+b_{2}+b_{3}>0 .
$$

For the triangular array $\{T(n, k)\}_{n \geq k \geq 0}$, we always assume that $T(0,0)>0$. Thus we have $T(n, k)>0$ for $0 \leq k \leq n$.

The following lemma is a special case of Theorem 2 of Kurtz [15].

Lemma 2.1 Suppose that the positive array $\{T(n, k)\}_{n \geq k \geq 0}$ satisfies the recurrence relation (2.2). Then, for given $n$, the sequence $\{T(n, k)\}_{0 \leq k \leq n}$ is log-concave, namely, for $0 \leq k \leq n$,

$$
T(n, k)^{2} \geq T(n, k-1) T(n, k+1) .
$$

Using the log-concavity (2.3) for the triangular array $\{T(n, k)\}_{n \geq k \geq 0}$, Liu and Wang obtained a sufficient condition for the polynomial sequence $\left\{P_{n}(q)\right\}_{n \geq 0}$ to be $q$-log-convex [16, Theorem 4.1].

Theorem 2.2 Suppose that the array $\{T(n, k)\}_{n \geq k \geq 0}$ of positive numbers satisfies the recurrence relation (2.2) and the additional condition

$$
\left(a_{2} b_{1}-a_{1} b_{2}\right) n+a_{2} b_{2} k+\left(a_{2} b_{3}-a_{3} b_{2}\right) \geq 0, \quad \text { for } 0<k \leq n .
$$

Then the polynomials $P_{n}(q)$ form a q-log-convex sequence.

This theorem can be used to show that the Bell polynomials and the Eulerian polynomials are $q$-log-convex. We will give alternative conditions for the recurrence relation (2.2) and will show that our conditions are satisfied by the Bell polynomials, the Bessel polynomials, the Ramanujan polynomials and the Dowling polynomials. It is also hoped that further studies will be carried out for more other types of recurrence relations with polynomial or even rational coefficients in $n$ and $k$.

An important property of the triangular array satisfying our conditions is described by the following lemma.

Lemma 2.3 Suppose that the array $\{T(n, k)\}_{n \geq k \geq 0}$ of positive numbers satisfies (2.2) with $a_{2}, b_{2} \geq 0$. Then, for any $l^{\prime} \geq l \geq 0$ and $m^{\prime} \geq m \geq 0$, we have

$$
T(m, l) T\left(m^{\prime}, l^{\prime}\right)-T\left(m, l^{\prime}\right) T\left(m^{\prime}, l\right) \geq 0 .
$$


Proof. Restate (2.4) as

$$
\frac{T\left(m, l^{\prime}\right)}{T(m, l)} \leq \frac{T\left(m^{\prime}, l^{\prime}\right)}{T\left(m^{\prime}, l\right)} .
$$

It suffices to show that for any $s \geq m$

$$
\frac{T\left(s, l^{\prime}\right)}{T(s, l)} \leq \frac{T\left(s+1, l^{\prime}\right)}{T(s+1, l)}
$$

Let

$$
f\left(l, l^{\prime}, s\right)=T(s, l) T\left(s+1, l^{\prime}\right)-T\left(s, l^{\prime}\right) T(s+1, l) .
$$

From the recurrence relation (2.2), we see that

$$
\begin{aligned}
& f\left(l, l^{\prime}, s\right)=\left(a_{1}(s+1)+a_{2} l^{\prime}+a_{3}\right) T\left(s, l^{\prime}\right) T(s, l) \\
& +\left(b_{1}(s+1)+b_{2} l^{\prime}+b_{3}\right) T\left(s, l^{\prime}-1\right) T(s, l) \\
& -\left(a_{1}(s+1)+a_{2} l+a_{3}\right) T(s, l) T\left(s, l^{\prime}\right) \\
& -\left(b_{1}(s+1)+b_{2} l+b_{3}\right) T(s, l-1) T\left(s, l^{\prime}\right) \\
& =\left(b_{1}(s+1)+b_{2} l^{\prime}+b_{3}\right) T\left(s, l^{\prime}-1\right) T(s, l) \\
& -\left(b_{1}(s+1)+b_{2} l+b_{3}\right) T(s, l-1) T\left(s, l^{\prime}\right) \\
& +a_{2}\left(l^{\prime}-l\right) T(s, l) T\left(s, l^{\prime}\right) \\
& \geq\left(b_{1}(s+1)+b_{2} l^{\prime}+b_{3}\right) T(s, l-1) T\left(s, l^{\prime}\right) \\
& -\left(b_{1}(s+1)+b_{2} l+b_{3}\right) T(s, l-1) T\left(s, l^{\prime}\right) \\
& +a_{2}\left(l^{\prime}-l\right) T(s, l) T\left(s, l^{\prime}\right) \\
& =b_{2}\left(l^{\prime}-l\right) T(s, l-1) T\left(s, l^{\prime}\right)+a_{2}\left(l^{\prime}-l\right) T(s, l) T\left(s, l^{\prime}\right) \text {, }
\end{aligned}
$$

Which is nonnegative in view of the condition $a_{2}, b_{2} \geq 0$. This completes the proof.

The main result of this paper is given below.

Theorem 2.4 Suppose that the array $\{T(n, k)\}_{n \geq k \geq 0}$ of positive numbers satisfies (2.2) with $a_{2}, b_{2} \geq 0$. Then the polynomial sequence $\left\{P_{n}(q)\right\}_{n \geq 0}$ is strongly q-log-convex, namely, for any $n \geq m \geq 1$,

$$
P_{m-1}(q) P_{n+1}(q)-P_{m}(q) P_{n}(q) \geq_{q} 0 .
$$

Proof. Throughout the proof, we simply write $P_{n}$ for $P_{n}(q)$. Let

$$
P_{n}^{\prime} P_{m-1}-P_{n} P_{m-1}^{\prime}=\sum_{i=0}^{m+n-1} A_{i} q^{i}
$$


where $P_{n}^{\prime}$ is the derivative of $P_{n}$ with respect to $q$. We claim that $A_{i} \geq 0$ for any $i$. Invoking the recurrence relation (2.2), the coefficient of $q^{i}$ in $P_{n}^{\prime} P_{m-1}$ equals

$$
\begin{aligned}
& \sum_{k=0}^{i}(i-k+1) T(n, i-k+1) T(m-1, k) \\
& =\sum_{k=0}^{i}(i-k+1)\left(a_{1} n+a_{2}(i-k+1)+a_{3}\right) T(n-1, i-k+1) T(m-1, k) \\
& \quad+\sum_{k=0}^{i}(i-k+1)\left(b_{1} n+b_{2}(i-k+1)+b_{3}\right) T(n-1, i-k) T(m-1, k) .
\end{aligned}
$$

Again, based on (2.2), the coefficient of $q^{i}$ in $P_{n} P_{m-1}^{\prime}$ equals

$$
\begin{aligned}
& \sum_{k=1}^{i+1} k T(n, i-k+1) T(m-1, k) \\
& =\sum_{k=1}^{i+1} k\left(a_{1} n+a_{2}(i-k+1)+a_{3}\right) T(n-1, i-k+1) T(m-1, k) \\
& \quad+\sum_{k=0}^{i} k\left(b_{1} n+b_{2}(i-k+1)+b_{3}\right) T(n-1, i-k) T(m-1, k)
\end{aligned}
$$

Let

$$
\begin{aligned}
c_{k} & =(i-2 k+1)\left(a_{1} n+a_{2}(i-k+1)+a_{3}\right) \\
d_{k} & =(i-2 k+1)\left(b_{1} n+b_{2}(i-k+1)+b_{3}\right)
\end{aligned}
$$

For $0 \leq k \leq\lfloor i / 2\rfloor$, we find that

$$
\begin{aligned}
c_{k}+c_{i-k+1}= & (i-2 k+1)\left(a_{1} n+a_{2}(i-k+1)+a_{3}\right) \\
& \quad+(2 k-i-1)\left(a_{1} n+a_{2} k+a_{3}\right) \\
= & a_{2}(i-2 k+1)^{2},
\end{aligned}
$$

which is nonnegative. Moreover,

$$
\begin{aligned}
d_{k}+d_{i-k}= & (i-2 k+1)\left(b_{1} n+b_{2}(i-k+1)+b_{3}\right) \\
& \quad+(2 k-i+1)\left(b_{1} n+b_{2}(k+1)+b_{3}\right) \\
= & b_{2}(i-2 k+1)(i-2 k)+2\left(b_{1} n+b_{2}(k+1)+b_{3}\right),
\end{aligned}
$$

which is also nonnegative.

By Lemma 2.3, for $0 \leq k \leq\lfloor i / 2\rfloor$, we obtain

$$
\begin{aligned}
T(m-1,0) T(n-1, i+1) & \geq T(m-1, i+1) T(n-1,0), \\
T(m-1, k) T(n-1, i-k+1) & \geq T(m-1, i-k+1) T(n-1, k), \\
T(m-1, k) T(n-1, i-k) & \geq T(m-1, i-k) T(n-1, k) .
\end{aligned}
$$


We now need to consider the parity of $i$. First, consider the case when $i$ is odd. Suppose that $i=2 j+1$ for some $j$. Clearly, we have $c_{j+1}=0$. Since all $T(n, k)$ are nonnegative, we get

$$
\begin{aligned}
A_{i}=( & +1)\left(a_{1} n+a_{2}(i+1)+a_{3}\right) T(m-1,0) T(n-1, i+1) \\
& -(i+1)\left(a_{1} n+a_{3}\right) T(m-1, i+1) T(n-1,0) \\
& +\sum_{k=1}^{i} c_{k} T(m-1, k) T(n-1, i-k+1) \\
& +\sum_{k=0}^{i} d_{k} T(m-1, k) T(n-1, i-k) \\
\geq & \sum_{k=1}^{i} c_{k} T(m-1, k) T(n-1, i-k+1) \\
& +\sum_{k=0}^{i} d_{k} T(m-1, k) T(n-1, i-k) \\
\geq & \sum_{k=1}^{j}\left(c_{k}+c_{i-k+1}\right) T(m-1, k) T(n-1, i-k+1) \\
& +\sum_{k=0}^{j}\left(d_{k}+d_{i-k}\right) T(m-1, k) T(n-1, i-k),
\end{aligned}
$$

which is nonnegative, since $c_{k}+c_{i-k+1} \geq 0$ and $d_{k}+d_{i-k} \geq 0$.

The case when $i$ is even can be dealt with via a similar argument. Suppose that $i=2 j$ for some $j$. In this case, we have $d_{j} \geq 0$. Therefore

$$
\begin{aligned}
A_{i} \geq \sum_{k=1}^{i} & c_{k} T(m-1, k) T(n-1, i-k+1) \\
& +\sum_{k=0}^{i} d_{k} T(m-1, k) T(n-1, i-k) \\
\geq & \sum_{k=1}^{j}\left(c_{k}+c_{i-k+1}\right) T(m-1, k) T(n-1, i-k+1) \\
& +\sum_{k=0}^{j-1}\left(d_{k}+d_{i-k}\right) T(m-1, k) T(n-1, i-k) \\
& +d_{j} T(m-1, j) T(n-1, j),
\end{aligned}
$$

which is nonnegative, since $c_{k}+c_{i-k+1} \geq 0, d_{k}+d_{i-k} \geq 0$ and $d_{j} \geq 0$.

Combining both cases, we are led to the assertion that $A_{i} \geq 0$, namely, 
for any $n \geq m \geq 1$,

$$
P_{n}^{\prime} P_{m-1}-P_{n} P_{m-1}^{\prime} \geq_{q} 0 .
$$

In view of the recurrence relation (2.2), we obtain

$$
\begin{aligned}
P_{m}= & \sum_{k=0}^{m} T(m, k) q^{k} \\
= & \sum_{k=0}^{m}\left(a_{1} m+a_{2} k+a_{3}\right) T(m-1, k) q^{k} \\
& \quad+\sum_{k=0}^{m}\left(b_{1} m+b_{2} k+b_{3}\right) T(m-1, k-1) q^{k} \\
= & \sum_{k=0}^{m-1}\left(a_{1} m+a_{2} k+a_{3}\right) T(m-1, k) q^{k} \quad(\text { by } T(m-1, m)=0) \\
& \quad+\sum_{k=1}^{m}\left(b_{1} m+b_{2} k+b_{3}\right) T(m-1, k-1) q^{k} \quad(\text { by } T(m-1,-1)=0) \\
= & \sum_{k=0}^{m-1}\left(a_{1} m+a_{2} k+a_{3}\right) T(m-1, k) q^{k} \\
& \quad+\sum_{k=0}^{m-1}\left(b_{1} m+b_{2}(k+1)+b_{3}\right) q T(m-1, k) q^{k} \\
= & \sum_{k=0}^{m-1}\left(a_{1} m+a_{3}\right) T(m-1, k) q^{k}+\sum_{k=0}^{m-1}\left(b_{1} m+b_{2}+b_{3}\right) q T(m-1, k) q^{k} \\
& \quad+\sum_{k=0}^{m-1}\left(a_{2}+b_{2} q\right) k T(m-1, k) q^{k} \\
= & \left(a_{1} m+a_{3}+b_{1} m q+b_{2} q+b_{3} q\right) P_{m-1}+\sum_{k=0}^{m-1}\left(a_{2} q+b_{2} q^{2}\right) k T(m-1, k) q^{k-1} \\
= & \left(a_{1} m+a_{3}+b_{1} m q+b_{2} q+b_{3} q\right) P_{m-1}+\left(a_{2}+b_{2} q\right) q P_{m-1}^{\prime},
\end{aligned}
$$

and hence

$$
P_{n+1}=\left(a_{1}(n+1)+a_{3}+b_{1}(n+1) q+b_{2} q+b_{3} q\right) P_{n}+\left(a_{2}+b_{2} q\right) q P_{n}^{\prime} .
$$

Substituting $P_{m}$ and $P_{n+1}$ into (2.5) yields

$$
\begin{aligned}
P_{m-1} P_{n+1}-P_{m} P_{n}= & \left(a_{1}+b_{1} q\right)(n-m+1) P_{m-1} P_{n} \\
& +q\left(a_{2}+b_{2} q\right)\left(P_{n}^{\prime} P_{m-1}-P_{n} P_{m-1}^{\prime}\right) .
\end{aligned}
$$

Since $a_{1}, a_{2}, b_{1}, b_{2} \geq 0$, the strong $q$-log-convexity of $\left\{P_{n}(q)\right\}_{n \geq 0}$ immediately follows from (2.9). 


\section{Applications}

In this section, we use Theorem 2.4 to show that the Bell polynomials, the Bessel polynomials, the Ramanujan polynomials and the Dowling polynomials are strongly $q$-log-convex.

\subsection{The Bell polynomials}

The Bell polynomials [1] are defined by

$$
B_{n}(q)=\sum_{k=0}^{n} S(n, k) q^{k},
$$

where $S(n, k)$ is the Stirling number of the second kind satisfying the following recurrence relation:

$$
S(n, k)=k S(n-1, k)+S(n-1, k-1), \quad n, k \geq 1
$$

with $S(0,0)=1$.

Corollary 3.1 The Bell polynomials are strongly q-log-convex.

Moreover, one can check that the following polynomials introduced by Tanny [21] are also strongly $q$-log-convex:

$$
F_{n}(q)=\sum_{k=0}^{n} k ! S(n, k) q^{k}
$$

\subsection{The Bessel polynomials}

The Bessel polynomials are defined by

$$
y_{n}(x)=\sum_{k=0}^{n} \frac{(n+k) !}{(n-k) ! k !}\left(\frac{x}{2}\right)^{k}
$$

and they have been extensively studied; See, for example, Burchnall [6], Carlitz [9], and Grosswald [12]. The Bessel polynomials satisfy the following recurrence relation [13]:

$$
y_{n}=(n x+1) y_{n-1}+x^{2} y_{n-1}^{\prime}
$$

Let

$$
T(n, k)=\frac{(n+k) !}{(n-k) ! k !} .
$$

From the recurrence (3.10), we deduce that

$$
T(n, k)=T(n-1, k)+(2 n+2 k-2) T(n-1, k-1), \quad n \geq k \geq 1 .
$$


Corollary 3.2 The Bessel polynomials are strongly q-log-convex.

\subsection{The Ramanujan polynomials}

The Ramanujan polynomials $R_{n}(x)$ are defined by the following recurrence relation:

$$
R_{1}(x)=1, \quad R_{n+1}(x)=n(1+x) R_{n}(x)+x^{2} R_{n}^{\prime}(x),
$$

where $R_{n}^{\prime}(x)$ is the derivative of $R_{n}(x)$ with respect to $x$. These polynomials are related to a refinement of Cayley's theorem due to Shor [19]. The connection between the Ramanujan polynomials and Shor's refinement of Cayley's formula was observed by Zeng [22]. Let $r(n, k)$ be the number of rooted labeled trees on $n$ vertices with $k$ improper edges. Shor [19] proved that $r(n, k)$ satisfies the following recurrence relation:

$$
r(n, k)=(n-1) r(n-1, k)+(n+k-2) r(n-1, k-1) .
$$

where $r(1,0)=1, n \geq 1, k \leq n-1$, and $r(n, k)=0$ otherwise. It can be seen from (3.11) and (3.12) that $R_{n}(x)$ is indeed the generating function of $r(n, k)$, namely,

$$
R_{n}(x)=\sum_{k=0}^{n} r(n, k) x^{k} .
$$

Dumont and Ramamonjisoa [11] independently found the same combinatorial interpretation for the coefficients of the Ramanujan polynomial $R_{n}(x)$.

Let $r^{\prime}(n, k)=r(n+1, k)$. Then the triangle $\left\{r^{\prime}(n, k)\right\}_{n \geq k \geq 0}$ satisfies the following recurrence relation

$$
r^{\prime}(n, k)=n r^{\prime}(n-1, k)+(n+k-1) r^{\prime}(n-1, k-1) .
$$

Corollary 3.3 The Ramanujan polynomials $R_{n}(q)$ are strongly q-log-convex.

\subsection{The Dowling polynomials}

The Dowling polynomials are defined as the generating functions of Whitney numbers of the second kind of Dowling lattices; See Benoumhani [2]. As a generalization of the partition lattice, Dowling [10] introduced a class of geometric lattices based on finite groups, called the Dowling lattice. Given a finite group $G$ of order $m \geq 1$, let $Q_{n}(G)$ be the Dowling lattice of rank $n$ associated to $G$, and, for $0 \leq k \leq n$, let $W_{m}(n, k)$ be the Whitney numbers 
of the second kind of $Q_{n}(G)$. The Dowling polynomial $D_{m}(n ; q)$ is defined by

$$
D_{m}(n ; q)=\sum_{k=0}^{n} W_{m}(n, k) q^{k}
$$

Benoumhani 2] also introduced the following generalized polynomials

$$
F_{m, 1}(n ; q)=\sum_{k=0}^{n} k ! W_{m}(n, k) m^{k} q^{k}, \quad F_{m, 2}(n ; q)=\sum_{k=0}^{n} k ! W_{m}(n, k) q^{k}
$$

Dowling [10] proved that the Whitney numbers $W_{m}(n, k)$ satisfy the following recurrence relation

$$
W_{m}(n, k)=(1+m k) W_{m}(n-1, k)+W_{m}(n-1, k-1), \quad n \geq k \geq 1,
$$

with the boundary conditions:

$$
\begin{aligned}
& W_{m}(n, n)=W_{m}(n, 0)=1, \text { for } n \geq 0, \\
& W_{m}(n, k)=0, \text { if } k>n .
\end{aligned}
$$

Note that, for $m=1$, the Whitney numbers $W_{m}(n, k)$ of the second kind are the Stirling numbers $S(n+1, k+1)$ of the second kind; See [10, 2].

From (3.14), Benoumhani [2] derived that

$$
F_{m, 2}(n ; q)=(q+1) F_{m, 2}(n-1 ; q)+q(q+m) F_{m, 2}^{\prime}(n-1 ; q),
$$

where $F^{\prime}$ is the derivative of $F$ with respect to $q$. Let $T(n, k)=k ! W_{m}(n, k)$. Then (3.15) implies that $T(n, k)$ satisfies the following recurrence relation

$$
T(n, k)=(1+m k) T(n-1, k)+k T(n-1, k-1), \quad n \geq k \geq 1 .
$$

Corollary 3.4 The Dowling polynomials $D_{m}(n ; q), F_{m, 1}(n ; q)$ and $F_{m, 2}(n ; q)$ are strongly q-log-convex.

\section{The Bessel transformation}

The objective of this section is to show that the Bessel transformation preserves log-convexity. The Bessel transformation is a linear transformation associated with the Bessel polynomials, which transforms a sequence $\left\{z_{n}\right\}_{n \geq 0}$ of nonnegative real numbers into a sequence $\left\{w_{n}\right\}_{n \geq 0}$ given by

$$
w_{n}=\sum_{k=0}^{n} \frac{(n+k) !}{(n-k) ! k !} z_{k}
$$


For a triangular array $\{T(n, k)\}_{n \geq k \geq 0}$, let $P_{n}(q)$ be the row generating function of $T(n, k)$. Given any $n \geq 1$, Liu and Wang defined the function $\alpha_{k}(n, i)$ for $0 \leq i \leq 2 n$ and $0 \leq k \leq\left\lfloor\frac{i}{2}\right\rfloor$. If $0 \leq k<\frac{i}{2}$, we have

$$
\begin{aligned}
\alpha_{k}(n, i)=T & (n-1, k) T(n+1, i-k) \\
& +T(n+1, k) T(n-1, i-k)-2 T(n, k) T(n, i-k) .
\end{aligned}
$$

If $i$ is even and $k=\frac{i}{2}$, then we have

$$
\alpha_{k}(n, i)=T(n-1, k) T(n+1, k)-T(n, k)^{2} .
$$

Liu and Wang [16] found the following connection between the $q$-log-convexity of $P_{n}(q)$ and the log-convexity preserving property of $T(n, k)$.

Theorem 4.1 ([16]) Suppose that the triangle $\{T(n, k)\}_{n \geq k \geq 0}$ of positive real numbers satisfies the following two conditions:

(C1) The sequence of polynomials $\left\{P_{n}(q)\right\}_{n \geq 0}$ is q-log-convex.

(C2) There exists an integer $k^{\prime}$ depending on $n$ and $i$ such that $\alpha_{k}(n, i) \geq 0$ for $k \leq k^{\prime}$ and $a_{k}(n, i)<0$ for $k>k^{\prime}$.

Then the linear transformation $w_{n}=\sum_{k=0}^{n} T(n, k) z_{k}$ preserves log-convexity.

We will use the above theorem to prove that the Bessel transformation preserves log-convexity. For any $n \geq 1$ and $0 \leq i \leq 2 n$, we introduce the following polynomials in $x$ :

$$
\begin{aligned}
& f_{1}(x)=(n+x+1)(n-i+x)(n+x)(n-i+x+1), \\
& f_{2}(x)=(n-x)(n+i-x+1)(n-x+1)(n+i-x), \\
& f_{3}(x)=(n-x+1)(n+i-x)(n+x)(n-i+x+1) .
\end{aligned}
$$

Let

$$
f(x)=f_{1}(x)+f_{2}(x)-2 f_{3}(x) .
$$

Lemma 4.2 For any $n \geq 1,0 \leq i \leq 2 n$ and $0 \leq k \leq\left\lfloor\frac{i}{2}\right\rfloor$, let

$$
\begin{aligned}
\beta_{k}(n, i)= & T(n+1, k) T(n-1, i-k)+T(n+1, i-k) T(n-1, k) \\
& -2 T(n, i-k) T(n, k),
\end{aligned}
$$

where

$$
T(n, k)=\frac{(n+k) !}{(n-k) ! k !}
$$

Then, for given $n$ and $i$, there exists an integer $k^{\prime}$ depending on $n$ and $i$ such that $\beta_{k}(n, i) \geq 0$ for $k \leq k^{\prime}$ and $\beta_{k}(n, i) \leq 0$ for $k>k^{\prime}$. 
Proof. Suppose that $n$ and $i$ are given. Clearly, if $k<i-n-1$, then $n<(i-k)-1$ and $\beta_{k}(n, i)=0$. If $k=i-n-1$, then

$$
\beta_{k}(n, i)=T(n+1, n+1) T(n-1, i-n-1) \geq 0 .
$$

Therefore, it suffices to determine the sign of $\beta_{k}(n, i)$ for $i-n-1<k \leq\left\lfloor\frac{i}{2}\right\rfloor$.

By (4.18), we have

$$
\begin{aligned}
\beta_{k}(n, i)= & \frac{(n+1+k) !}{(n+1-k) ! k !} \times \frac{(n-1+i-k) !}{(n-1-i+k) !(i-k) !} \\
& \quad+\frac{(n+1+i-k) !}{(n+1-i+k) !(i-k) !} \times \frac{(n-1+k) !}{(n-1-k) ! k !} \\
& \quad-\frac{2(n+i-k) !}{(n-i+k) !(i-k) !} \times \frac{(n+k) !}{(n-k) ! k !} \\
= & \left(\frac{(n+k+1)(n-i+k)}{(n-k+1)(n+i-k)}+\frac{(n-k)(n+i-k+1)}{(n+k)(n-i+k+1)}-2\right) \\
& \quad \times \frac{(n+i-k) !}{(n-i+k) !(i-k) !} \times \frac{(n+k) !}{(n-k) ! k !} \\
= & \frac{f(k) T(n, i-k) T(n, k)}{f_{3}(k)} .
\end{aligned}
$$

Note that for $n \geq 1,0 \leq i \leq 2 n$ and $i-n-1<k \leq\left\lfloor\frac{i}{2}\right\rfloor$, we have

$$
f_{3}(k)>0, \quad T(n, i-k) T(n, k) \geq 0 .
$$

By the definition (4.17) of $f(x)$, we find

$$
f^{\prime}(x)=2(2 x-i) g(x),
$$

where

$$
g(x)=2\left(2+8 n^{2}-i+8 n\right) .
$$

Thus, for $i \leq 2 n$ and $x \leq \frac{i}{2}$, we have

$$
g(x) \geq 2\left(2+8 n^{2}+6 n\right)>0, \quad f^{\prime}(x) \leq 0 .
$$

Therefore, $f(x)$ is decreasing in $x$ on the interval $\left(-\infty, \frac{i}{2}\right]$. This implies that there exists an integer $k^{\prime}$ such that $\beta_{k}(n, i) \geq 0$ for $k \leq k^{\prime}$ and $\beta_{k}(n, i) \leq 0$ for $k>k^{\prime}$.

Combining Corollary [3.2, Lemma 4.2 and Theorem 4.1, we deduce the following theorem. 
Theorem 4.3 If $\left\{z_{k}\right\}_{k \geq 0}$ is a log-convex sequence of positive real numbers, then the sequence $\left\{w_{n}\right\}_{n \geq 0}$ defined by

$$
w_{n}=\sum_{k=0}^{n} \frac{(n+k) !}{(n-k) ! k !} z_{k}
$$

is also log-convex.

Acknowledgments. This work was supported by the 973 Project, the PCSIRT Project of the Ministry of Education, the Ministry of Science and Technology, and the National Science Foundation of China.

\section{References}

[1] E.T. Bell, Exponential Polynomials, Ann. Math. 35 (1934), 258-277.

[2] M. Benoumhani, On some numbers related to Whitney numbers of Dowling lattices, Adv. Appl. Math. 19 (1997), 106-116.

[3] B.C. Berndt, Ramanujan's Notebooks, Part I, Chap. 3, Springer-Verlag, New York, 1985.

[4] F. Brenti, Unimodal, log-concave, and Pólya frequency sequences in combinatorics, Mem. Amer. Math. Soc. 413 (1989), 1-106.

[5] F. Brenti, Log-concave and unimodal sequences in algebra, combinatorics and geometry: an update, Contemp. Math. 178 (1994), 71-89.

[6] J.L. Burchnall, The Bessel polynomials, Canad. J. Math. 3 (1951), 6268.

[7] L. M. Butler, The $q$-log concavity of $q$-binomial coefficients, J. Combin. Theory Ser. A 54 (1990), 54-63.

[8] L. M. Butler, W. P. Flanigan, A note on log-convexity of $q$-Catalan numbers, arXiv:math/0701065.

[9] L. Carlitz, A note on the Bessel polynomials, Duke. Math. J. 23 (1957), $151-162$.

[10] T.A. Dowling, A class of geometric lattices based on finite groups, J. Combin. Theory Ser. B 14 (1973), 61-86; Erratum, J. Combin. Theory Ser. B 15 (1973), 211.

[11] D. Dumont, A. Ramamonjisoa, Grammaire de Ramanujan et arbres de Cayley, Electron. J. Combin. 3(2) (1996), R17. 
[12] E. Grosswald, On some algebraic properties of the Bessel polynomials, Trans. Amer. Math. Soc. 71 (1951), 197-210.

[13] H.L. Krall, O. Frink, A new class of orthogonal polynomials: The Bessel polynomials, Trans. Amer. Math. Soc. 65 (1949), 100-115.

[14] C. Krattenthaler, On the $q$-log-concavity of Gaussian binomial coefficients, Monatsh. Math. 107 (1989), 333-339.

[15] D.C. Kurtz, A note on concavity properties of triangular arrays of numbers, J. Combin. Theory Ser. A 13 (1972), 135-139.

[16] L.L. Liu and Y. Wang, On the log-convexity of combinatorial sequences, to appear in Adv. Appl. Math., arXiv:math.CO/0602672.

[17] P. Leroux, Reduced matrices and $q$-log concavity properties of $q$-Stirling numbers, J. Combin. Theory Ser. A 54 (1990), 64-84.

[18] B. E. Sagan, Log concave sequences of symmetric functions and analogs of the Jacobi-Trudi determinants, Trans. Amer. Math. Soc. 329 (1992), 795-811.

[19] P. Shor, A new proof of Cayley's formula for counting labeled trees, J. Combin. Theory Ser. A 71 (1995), 154-158.

[20] R. P. Stanley, Log-concave and unimodal sequences in algebra, combinatorics and geometry, Ann. New York Acad. Sci 576 (1989), 500-535.

[21] S. Tanny, On some numbers related to the Bell numbers, Canad. Math. Bull. 17 (1975), 733-738.

[22] J. Zeng, A Ramanujan sequence that refines the Cayley formula for trees, Ramanujan J. 3 (1999), 45-54. 